\title{
Minimizing Morbidity while Preserving Outcome after Inguinal Lymphadenectomy: Navigating between Scylla and Charybdis
}

\author{
Vernon K. Sondak, MD ${ }^{1,2,3}$ and Amod A. Sarnaik, $M D^{1,2}$ \\ ${ }^{1}$ Department of Cutaneous Oncology, Moffitt Cancer Center, Tampa, FL; ${ }^{2}$ Department of Oncologic Sciences, University \\ of South Florida College of Medicine, Tampa, FL; ${ }^{3}$ Department of Surgery, University of South Florida College of \\ Medicine, Tampa, FL
}

Radical lymphadenectomy for microscopic or macroscopic metastases from cutaneous melanoma is associated with cure rates higher than many other widely performed radical procedures, such as radical pancreaticoduodenectomy or esophagectomy, that are associated with higher morbidity and mortality rates. ${ }^{1}$ Nonetheless, the morbidity associated with lymphadenectomy, especially inguinal or ilioinguinal lymphadenectomy, leads some patients to refuse - and even leads many surgical oncologists to discourage their patients from undergoing - this potentially curative procedure. However, the past few decades have seen substantial advances in our ability to identify the patients who are most appropriate for inguinal or ilioinguinal lymphadenectomy. These advances include the widespread adoption of sentinel node biopsy to identify microscopic nodal metastases, and the use of ultrasound and PET scanning to identify macroscopic nodal metastases before they become palpable or symptomatic. Whereas earlier detection in and of itself can lower operative morbidity, there has been much less attention paid to decreasing the morbidity of inguinal lymphadenectomy by altering the actual surgical technique. Fortunately for our patients, this has begun to change. In this issue of the Annals of Surgical Oncology, two articles describe efforts to decrease the morbidity of inguinal node dissection by performing the procedure through less invasive approaches than typically employed. ${ }^{2,3}$ Before we discuss these two papers, however, an examination of what is already known about the morbidity of inguinal node dissection is in order.

(C) Society of Surgical Oncology 2011

Published Online: 19 January 2011

V. K. Sondak, MD

e-mail: vernon.sondak@moffitt.org
Inguinal node dissection is associated with substantive short-term and chronic morbidity. ${ }^{4}$ Wound complications and prolonged lymphatic drainage are common short-term problems, whereas deep venous thrombosis is rare but potentially life-threatening. In prospective data collected from 127 patients who underwent complete inguinal lymphadenectomy as part of the Sunbelt Melanoma Trial, $51.2 \%$ developed a significant postoperative complication. ${ }^{5}$ Minimizing acute complications begins with the initial diagnosis of metastatic disease. The sentinel node biopsy site should be positioned and oriented to facilitate using the smallest possible incision should a subsequent inguinal lymphadenectomy prove necessary, and we strongly believe that the groin crease itself should be avoided for sentinel node biopsy incisions so that the lymphadenectomy incision need not involve or cross the groin. ${ }^{4}$ Equally importantly, when palpable nodes are encountered in the groin, fine-needle aspiration should be utilized to establish the diagnosis, because open biopsy is associated with higher acute morbidity during the subsequent lymphadenectomy. ${ }^{6}$ For all the consternation surgeons show when primary melanomas are diagnosed by the expedient shave biopsy, we should be collectively ashamed at how routinely we employ open node biopsy when needle aspiration would suffice and be far better for the patient. In the same vein, although preoperative node ultrasonography has to date not been shown to be a satisfactory substitute for sentinel node biopsy on a routine basis, ${ }^{7,8}$ we do use preoperative inguinal nodal ultrasonography for our patients with T4 lesions. In the event of detecting an abnormal node, this is followed by fine-needle aspiration biopsy to allow for a one-stage procedure combining wide excision and lymphadenectomy, eliminating the sentinel node biopsy incision entirely in a small subset of patients at high risk of needing a lymphadenectomy. ${ }^{9}$ 
Even more important than how metastatic melanoma in an inguinal node is diagnosed is when it is diagnosed; accumulating data strongly suggest that the morbidity of inguinal node dissection is substantially less when the procedure is performed for microscopic rather than macroscopic disease. ${ }^{10,11}$ Faries et al., reporting prospective data from the first randomized Multicenter Selective Lymphadenectomy Trial (MSLT-1), found that patients who underwent lymphadenectomy at any site after a positive sentinel node biopsy had a statistically significantly lower rate of lymphedema than those who underwent lymphadenectomy after a palpable nodal recurrence (12.4 vs. $20.4 \%$; $P=0.04) .{ }^{11}$ For patients undergoing inguinal or ilioinguinal lymphadenectomy, the corresponding lymphedema rates were 21.9 vs. $34.7 \%$ for early or delayed procedures. Interestingly, contrary to widespread belief, lymphedema rates did not differ for inguinal and ilioinguinal dissections (21.4 vs. $22.6 \%$, early, and 36.4 vs. $34.2 \%$, delayed). One note of caution regarding any attempt to quantify the frequency of lymphedema after inguinal lymphadenectomy: standardized evaluation and reporting is lacking, and rates vary widely depending on how lymphedema is defined and how carefully it is sought. ${ }^{12}$ As an illustration, in the Sunbelt Melanoma Trial, where all lymphadenectomies were performed for sentinel-node positive disease, the reported lymphedema rate for inguinal lymphadenectomies was $31.5 \%$ in contrast to the $21.9 \%$ rate from MSLT- $1 .{ }^{5}$

If the timing and method of diagnosing inguinal metastases are so important, what if any contributions are made by the technique of conducting the lymphadenectomy itself? First and foremost, the most feared sequela of lymphadenectomy should be recurrence of the tumor within the nodal basin, because that represents failure to achieve the single overarching goal of performing the procedure in the first place. Any modification to the procedure, including omitting it altogether for selected patients, needs to be evaluated in that light. We also should be cognizant of the fact that the "noninferiority" of a modified or averted procedure can only be convincingly demonstrated in a large, randomized trial with substantial follow-up. Neither of the experiences with modified node dissections described in this issue were randomized, and both had relatively short follow-up. Still, we must start somewhere if we are to make progress toward eliminating morbidity as a deterrent to undergoing a potentially curative procedure.

Spillane et al. explored whether two relatively short incisions that avoid the inguinal crease allow performance of a technically satisfactory dissection with acceptable oncologic outcomes and morbidity. ${ }^{3}$ Pursuant to the issues raised above, this study is nonrandomized and comprised relatively few patients $(n=20)$ undergoing this "minimal access" approach with short (5 months) follow-up. In their experience, the minimal access approach resulted in similar lymph node yield (recognizing that "optimal" and "minimum" inguinal node yields remain to be conclusively defined ${ }^{13}$ ) and complication rates to those expected from the traditional approach, suggesting that the limited incisions did not hamper the conduct of the surgery. This hardly seems surprising, because with appropriate retraction and gentle handling of flaps that are made no wider or thinner than necessary, one can perform the inguinal portion of the dissection entirely through a suprainguinal incision. ${ }^{4} \mathrm{We}$ frequently do this for node-positive patients whose biopsy site (sentinel, open, or needle) is situated superior to the inguinal ligament. In our experience, a second small incision near the apex of the femoral trial may be necessary for the rare patient who has a long, narrow femoral triangle, especially to identify the saphenous vein distally in the wound. With a single suprainguinal incision, we rarely mobilize the sartorius muscle, and often preserve the saphenous vein in patients with micrometastases if the vein and saphenofemoral junction are not adherent or adjacent to the biopsy cavity. ${ }^{4}$

More innovative-and controversial-is Delman's approach of videoscopic inguinal lymphadenectomy. This procedure adheres more closely to principles of videoassisted or endoscopic minimally invasive procedures in the abdomen and chest, but does not adhere to the same oncologic principles as the open radical inguinal lymphadenectomy, by virtue of leaving the node biopsy site in place. ${ }^{2}$ We have serious reservations about leaving the sentinel lymph node scar intact, and at present we cannot condone this approach when macroscopic nodal disease has been removed by an open biopsy-particularly in the face of extracapsular extension or when the original biopsy was performed piecemeal. There is as yet inadequate corroborating experience from other series, and too short of followup in this series, to determine the consequence of leaving the node biopsy scar in situ. Conversely, there is a significant historical parallel to laparoscopic colon and gallbladder resections for cancer, where port site recurrences occurred at high frequencies in cases where oncologic principles were not followed. ${ }^{14,15}$ This is no small point; if retaining the scar increases the regional failure rate, enthusiasm for this procedure would be minimal, whereas routinely removing the scar would reduce most of the advantage of the videoscopic approach. The total length of inguinal incisions made when the biopsy site is excised and ports are placed would nearly equal the length of the single skin incision that we make currently for our standard lymphadenectomy after sentinel node biopsy (typically only $2-3 \mathrm{~cm}$ longer than the length of the sentinel node biopsy scar). ${ }^{4}$ Until and unless the safety of leaving the node biopsy incision in place can be validated, it may be that this videoscopic approach should be reserved for patients in whom the diagnosis of nodal metastasis has been made by fine or core needle biopsy. 
In other considerations, an open inguinal lymphadenectomy can be performed currently much more quickly than described for the initial experience with the videoscopic approach (albeit that time is sure to decrease as experience builds). The median operative time in Delman's series was $2.75 \mathrm{~h}$, whereas we expect the inguinal lymphadenectomy specimen to be removed within 60 to 70 min after the skin incision in the open version, even if performed through a limited skin incision. Sartorius transposition is routinely omitted in the videoscopic version, but how often it is needed in the open procedure-and how much morbidity it adds-are unsettled issues at present. Finally, length of stay, which should rarely be the sole criterion for accepting a minimally invasive modification of a standard operation because it is so dependent on nonoperative factors, of the videoscopic version (median 1 day; mean 3 days) seems outstanding compared with the MSLT-1 experience (10.2 days for patients who undergo inguinal dissections and 13.9 days for ilioinguinal dissections). ${ }^{11}$ However, MSLT-1 closed to accrual more than 8 years ago, and most patients were enrolled outside the United States. So, is this representative of a contemporary U.S. result? We think not; for the past 6 years we have kept inguinal node dissection patients in the hospital for only 1 day and ilioinguinal dissection patients for 2 to 3 days. Also, whereas indications for pelvic node dissection remain ill-defined, if an external iliac and obturator node dissection is added to the videoscopic inguinal procedure, it remains unclear how much, if any, advantage of the minimally invasive approach would persist.

In the final sentence of their abstract, Delman et al. indicate that a randomized trial comparing open and videoscopic inguinal lymphadenectomy is currently in progress. Although it has almost become a cliché to state that randomized trials are needed before a new technique can be adopted, this begs the question of whether we are ready for a randomized trial. Specifically, do we know what would be the most appropriate endpoint of such a trial? There are several possibilities, including survival, recurrence, cost, and lymphedema rates. Who would perform the trial? It would not be fair to conduct the trial with surgeons who have not completed the learning curve for videoscopic lymphadenectomy, so the techniques would need to be implemented at several centers before a largescale trial can commence. Finally, we would all benefit from a better understanding of the actual morbidity, length of stay, and regional recurrence rates of open inguinal lymphadenectomy as currently performed at major melanoma centers. ${ }^{12}$

As surgical oncologists, we have always tried to steer our patients clear of regional recurrence, but we also fear the short- and long-term morbidity that radical lymphadenectomy conveys. We face a unique opportunity to reevaluate our position and perhaps chart an entirely new course to limit morbidity while preserving oncologic outcomes. Regardless of whether either of the less invasive approaches described in this issue of the Annals are ultimately adopted, there is no doubt that we and our patients will benefit from the spotlight that they shine on the morbidity of inguinal lymphadenectomy, and the things we can do as surgeons to navigate our patients away from harm.

\section{REFERENCES}

1. McMasters KM. Why does no one want to perform lymph node dissection anymore? Ann Surg Oncol. 2010;17:358-61.

2. Delman KS, Kooby DA, Rizzo M, Ogan K, Master V. Initial experience with videoscopic inguinal lymphadenectomy. Ann Surg Oncol. 2010. doi:10.1245/s10434-010-1490-5.

3. Spillane AJ, Tucker M, Pasquali S. A pilot study reporting outcomes for melanoma patients of a minimal access ilio-inguinal dissection technique based on two incisions. Ann Surg Oncol. 2010. doi:10.1245/s10434-010-1455-8.

4. Sarnaik AA, Puleo CA, Zager JS, Sondak VK. Limiting the morbidity of inguinal lymphadenectomy for metastatic melanoma. Cancer Ctrl. 2009;16:240-7.

5. Wrightson WR, Wong SL, Edwards MJ, Chao C, Reintgen DS, Ross MI, et al. Complications associated with sentinel lymph node biopsy for melanoma. Ann Surg Oncol. 2003;10:676-80.

6. Nathansohn N, Schachter J, Gutman H. Patterns of recurrence in patients with melanoma after radical lymph node dissection. Arch Surg. 2005;140:1172-7.

7. Sanki A, Uren RF, Moncrieff M, Tran KL, Scolyer RA, Lin HY, et al. Targeted high-resolution ultrasound is not an effective substitute for sentinel lymph node biopsy in patients with primary cutaneous melanoma. J Clin Oncol. 2009;27:5614-9.

8. Chai CY, Zager JS, Marzban SS, Rossi RM, Szabunio M, Sondak VK. Preoperative ultrasound is not useful for identifying nodal metastasis in melanoma patients undergoing sentinel lymph node biopsy. Ann Surg Oncol. 2010;17:S106.

9. Van Akkooi ACJ, Voit CA, Verhoef C, Eggermont AMM. Potential cost-effectiveness of US-guided FNAC in melanoma patients. Ann Surg Oncol. 2010;17:660-2.

10. Sabel MS, Griffith KA, Arora A, Shargorodsky J, Blazer DG 3rd, Rees R, et al. Inguinal node dissection for melanoma in the era of sentinel lymph node biopsy. Surgery. 2007;141:728-35.

11. Faries MB, Thompson JF, Cochran A, Elashoff R, Glass EC, Mozzillo N, et al. The impact of morbidity and length of stay of early versus delayed complete lymphadenectomy in melanoma: results of the Multicenter Selective Lymphadenectomy Trial (I). Ann Surg Oncol. 2010;17:3324-9.

12. Chang SB, Askew RK, Xing Y, Weaver S, Gershenwald JE, Lee JE, et al. Prospective assessment of postoperative complications and associated costs following inguinal lymph node dissection (ILND) in melanoma patients. Ann Surg Oncol. 2010;17:2764-72.

13. Spillane AJ, Cheung BL, Stretch JR, Scolyer RA, Shannon KF, Quinn MJ, et al. Proposed quality standards for regional lymph node dissections in patients with melanoma. Ann Surg. 2009;249:473-80.

14. Bonnor RM, Ludwig KA. Laparoscopic colectomy for colon cancer: comparable to conventional oncologic surgery? Clin Colon Rectal Surg. 2005;18:174-81.

15. Goetze TO, Paolucci V. Use of retrieval bags in incidental gallbladder cancer cases. World J Surg. 2009;33:2161-5. 